ARTICLE

\title{
Dichotomaria marginata (Rhodophyta) as a bioindicator for marine pollution: An overview about its metabolites and adsorbed pollutants
}

\author{
Dichotomaria marginata (Rhodophyta) como bioindicador para la contaminación \\ marina: Una visión general sobre sus metabolitos y contaminantes adsorbidos

\section{Angelica Nunes Garcia ${ }^{1 *}$, Erika Mattos Stein ${ }^{2}$, Leonardo Zambotti Villela ${ }^{2}$, Nair S. Yokoya $^{1}$, Pio Colepicolo Neto ${ }^{2}$ and Luciana Retz de Carvalho ${ }^{1}$}

\author{
${ }^{1}$ Núcleo de Pesquisa em Ficologia, Instituto de Botânica de São Paulo, SP, Brazil \\ ${ }^{2}$ Departamento de Bioquímica, Instituto de Química, Universidade de São Paulo, SP, Brazil \\ *Corresponding author: angelsgarcia@uol.com.br
}

\begin{abstract}
Resumen.- Las macroalgas se consideran bioindicadores de la contaminación marina, ya que tienen la capacidad de reaccionar rápidamente a los cambios en su entorno. En consecuencia, las poblaciones de macroalgas fluctúan, según las características de las especies y las estrategias de adaptación. Sus polisacáridos de la pared celular contienen grupos sulfato que son capaces de retener y acumular metales pesados. Además de los contaminantes tradicionales, los contaminantes emergentes están siendo reconocidos en ambientes acuáticos. En este trabajo, los contaminantes emergentes se han identificado después de ser desorbidos de la macroalga, Dichotomaria marginata, recolectada en la playa de Fortaleza, Ubatuba - SP, Brasil. Basado en el hecho de que las redes de polisacáridos de algas tienen el potencial de formar enlaces de hidrógeno con compuestos polares, se planteó la hipótesis de que estos contaminantes estarían unidos a polímeros de azúcar. Los compuestos presentes en las muestras de $D$. marginata se identificaron mediante cromatografía de gases y líquidos-espectrometría de masas (GC-MS y LC-MS), asistidos por métodos computacionales. Fue posible identificar inequívocamente 22 contaminantes emergentes con GC-MS y 16 sustancias con LC-MS.
\end{abstract}

\begin{abstract}
Palabras clave: Contaminantes marinos emergentes, polisacáridos de algas, carbonato de calcio, desorbancia, macroalgas, bioindicadores

Abstract.- Macroalgae are considered bioindicators for marine pollution, because they have the ability to quickly react to changes in their environment. In consequence, macroalgae populations fluctuate, according to species characteristics and adaptive strategies. Their cell wall polysaccharides contain sulfate groups that are capable of retaining and accumulating heavy metals. In addition to traditional contaminants, emerging pollutants are being recognized in aquatic environments. Herein, emerging pollutants have been identified after being desorbed from the macroalga Dichotomaria marginata, collected from Fortaleza Beach, Ubatuba - SP, Brazil. Based on that algal polysaccharide networks have the potential of forming hydrogen bonds with polar compounds, it was hypothesized that these pollutants would be bound to sugar polymers. Compounds present in the $D$. marginata samples were identified using both gas and liquid chromatography/mass spectrometry (GC/MS and HPLC/MS), assisted by computational methods. It was possible to unequivocally identify 22 emerging contaminants with GC/MS, and 16 substances with HPLC/MS.
\end{abstract}

Key words: Emerging marine contaminants, algal polysaccharides, calcium carbonate, desorbance, macroalgae, bioindicators

\section{INTRODUCTION}

Over a century ago it was first proposed that there is a connection between the presence, absence or abundance of algal species and the characteristics of the marine environment (Kolkwitz \& Marsson 1908). In fact, many species of algae have adapted to eutrophic conditions by undergoing morphological, physiological and/or ecological changes, which promote the growth of the population (Omar 2010), and the disappearance of others coincides with habitat eutrophication (Sousa \& Cocentino 2004, Holt \& Miller 2010, Omar 2010).
Previous studies have demonstrated that algae have the ability to incorporate heavy metals and nutrients (Omar 2010, Rajfur 2014, Rajfur \& Kłos 2014). This characteristic is attributed to the high adsorption power of the thallus, whose cell walls are made up of polysaccharides, proteins, and lipids.

The polysaccharide composition of the cell walls and the of the intercellular matrix can differ in different groups of red algae, but related to storage carbohydrates, there are basically two types of them (floridosides and digeneaside). 
The floridoside [2-O-a-D-galactopyranosylglycerol, (Fig. 1a)] is the prevalent one. Besides that, there are also two diastereoisomeric isofloridosides, $\{[1-\mathrm{O}-\mathrm{a}-\mathrm{D}-$ galactopyranosyl-D-glycerol (Fig. 1b)] and [1-O-a-Dgalactopyranosyl-L-glycerol (Fig. 1c)]\}. The digeneaside (2-O-a-D-mannopyranosyl-D-glyceric acid) is represented by the formula depicted in Figure 1d. D. marginata also biosynthesize specific water-soluble structural polysaccharides: sulfated xylomannans and neutral xylans (Usov 2011).

These polysaccharides contain a large amount of hydroxyl groups, which endows these carbohydrates with a hydrophilic character and the ability to form hydrogen bonds with environmental molecules. Hydrogen bonds occur when the hydrogen atom bound covalently to an electronegative atom (hydrogen donor group) electrostatically interacts with electronegative atoms (hydrogen receptor) forming non-covalent bonds. In the hydrogen donor group, usually the electronegative atom is either oxygen, nitrogen or sulfur. Hydrogen receptors are typically O, N, S and also phenols (Schaeffer 2008).

The lipids contain carboxylate, sulfate and phosphate anionic groups, which function as metal-binding sites, as well as positively charged groups. Additionally, environmental $\mathrm{pH}$ and the presence of competing ions can impact the adsorption process (Gadd 2009, Chopra \& Pathak 2010). Algae also adsorb pollutants through the use of phytochelatins, which are peptides that are capable of chelating heavy metals (Inouhe 2005).

a)

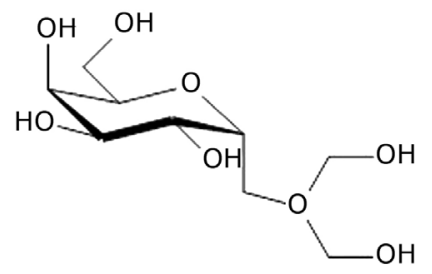

c)

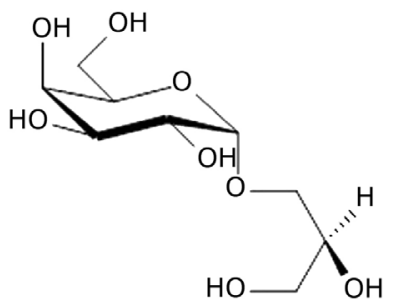

According to Dokulil (2003), the pollutant adsorption process occurs in two steps. In the first step, the pollutant accumulates, by passive adsorption, on the outer surface of the seaweed thallus. In the second step, these compounds/ substances are then slowly adsorbed, by metabolic processes. Interestingly, previous studies have demonstrated that these macroalgae can remove alkyl benzene sulfonates, phthalates, and textile dyes from aqueous solutions (Fernandez et al. 1995, El-Maghraby 2013), thus emphasizing the adsorptive capabilities of these organisms.

In the marine environment, all organisms are in constant contact with dissolved or suspended substances. For example, in addition to common salts, bases, and organic or inorganic acids, marine organisms are also in contact with halogens, alkali metals, oxygen, nitrogen, silicon, trace elements, organic compounds (produced by aquatic organisms themselves) and pollutants (Bryan 1979, Millero et al. 2008).

Among these pollutants are emerging contaminants, which are a group of substances used extensively in everyday life. These contaminants are not currently included in routine water monitoring programs, norms or legislation for environmental control (Calvo-Flores et al. 2018). This is primarily because the existing toxicity data are insufficient for establishing the respective reference doses. As a consequence, these unregulated pollutants pose a serious challenge to water quality regulators (Barceló 2003, Farré et al. 2008, Sousa et al. 2018). Furthermore, these agents have been detected in numerous aquatic organisms (Mearns et al. 2015).

b)

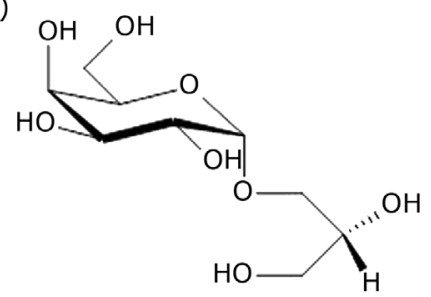

d)

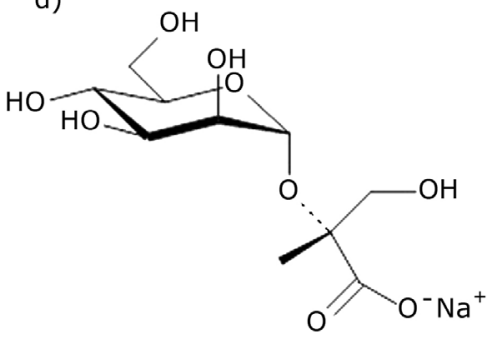

Figure 1. Natural storage carbohydrates biosynthesized by red algae. a-c) Floridoside and d) digeneaside (figure modified from Usov et al. 1981) / Carbohidratos de almacenamiento natural biosintetizados por algas rojas. a-c) Floridosido y d) digeneasida (figura modificada de Usov et al. 1981) 
With regards to macroalgae, compounds such as: surfactants, phthalates and polychlorinated biphenyls have been isolated from their extracts (Mackintosh et al. 2004, Gressler et al. 2012, Osman et al. 2013, Shobier et al. 2016, Stranska-Zachariasova et al. 2017). These three groups of compounds have antifungal activity (Uyanik et al. 2009, Memić et al. 2011, Lotfy et al. 2018, Paluch et al. 2018, Fait et al. 2019). In the late 1970s, phthalates were identified as algal components (Noguchi et al. 1979), and the bioconcentration, as well as the trophic magnification in the aquatic food web have been evaluated (Mackintosh et al. 2004).

In studies with algae, gas chromatography/mass spectrometry (GC/MS) has been utilized for the detection of phthalates, adipates and surfactants, due to the inherent thermal stability, volatility and of low polarity of these compounds (Mackintosh et al. 2004, Gressler et al. 2012, Osman et al. 2013, Shobier et al. 2016, StranskaZachariasova et al. 2017). On the other hand, high performance liquid chromatography coupled with mass spectrometry (HPLC/MS) has been successfully employed in research on non-volatile, thermally unstable and more polar contaminants (Goulitquer et al. 2012).

Both analytical platforms (GC/MS and HPLC/MS) become more valuable when assisted by computer tools such as the AMDIS (Automated Mass Spectrometry Deconvolution and Identification System) algorithm, which can separate co-eluting components (deconvolution), provide accurate molecular weights and generate both qualitative and quantitative data for each substance (Du \& Zeisel 2013).

The search for biological activities in algae extracts whether they are polar or nonpolar - is the starting point for the discovery and isolation of substances with interesting biological actions. Regarding antifungal activity, it was detected in the hexane extracts of Dictyota dichotoma, Dictyota dichotoma var. implexa and Dilophus spiralis (Moreau et al. 1988) and of Laurencia species (Stein et al. 2011) and Hydroclathrus clathratus (Vimala \& Poonghuzhali 2017) and in the dichloromethane extracts of Ceramium rubrum (Cortés et al. 2014).

Thus, the present study was designed to identify metabolites from the algae and potential emerging pollutants, desorbed from the thallus of the benthic marine macroalgae Dichotomaria marginata (J. Ellis \& Solander) Lamark, 1816, which may have antifungal activity, using a combination of GC/MS and HPLC/MS, followed by spectral deconvolution. The results will demonstrate the utility of $D$. marginata as a bioindicator for emerging contaminants and the interference of some of these contaminants in biological activity assays of crude extracts.

\section{Materials AND METHOdS}

\section{SAMPLE COLLECTION AND EXTRACTION}

Dichotomaria marginata, previously named as Galaxaura marginata, is a calcareous alga that belongs to the Rhodophyta (Johnson et al. 2014). Samples of this species were manually collected from Fortaleza Beach, in Ubatuba, São Paulo, Brazil (2350'15”S / 40¹7'40”W), during low tides. A voucher specimen (SP 428.540) was deposited at the Maria Eneyda P. Kauffman Fidalgo Herbarium, Instituto de Botânica, São Paulo.

Following collection, samples were washed, frozen and transported inside thermal box with ice to the laboratory. At the laboratory, they were shade dried at room temperature (20-25 ${ }^{\circ} \mathrm{C}$ ), and ground into fine particles (Carvalho et al. 2003). The powdered biomass (419.63 g) was extracted with hexane $(5 \times 3.45 \mathrm{~L})$ until exhaustion; the pooled extracts were filtered through filter paper (Whatman ${ }^{\circledR}$ No. 5), the solvent was removed under reduced pressure and the final product was weighed (Bhagavathy et al. 2011).

\section{HEXANE EXTRACT FRACTIONATION}

A portion (130 g) of the extract was subjected to column chromatography (designated as C-I) on silica gel (60, 0.2$0.5 \mathrm{~mm}$, Vetec), and eluted with $400 \mathrm{~mL}$ of each of the following solutions: dichloromethane (DCM)/methanol $(\mathrm{MeOH})$ 99.5:0.5 v/v, DCM/MeOH 97.3:2.7 v/v, DCM/ $\mathrm{MeOH}$ 95:5 v/v and $\mathrm{MeOH}$ 100\%. Each eluted $10 \mathrm{~mL}$ fraction was dried under an air stream, and subjected to Planar Chromatography (PC), using vanillin-sulfuric acid as visualization reagent. Similar fractions were pooled together.

\section{RE-FRACTIONATION OF POOLED FRACTIONS FROM C-I}

Combined fractions from C-I (Fractions 4-9) (53.4 mg) were refractionated on a silica gel column (designated as C-II), using DCM/MeOH 99.5:0.5 v/v as the mobile phase, which yielded 55, $1 \mathrm{~mL}$, fractions. After PC analysis, similar fractions were combined. The combined fractions from both columns (C-I and C-II) were then analyzed by $\mathrm{GC}$ / MS and HPLC/MS. 


\section{GAS CHROMATOGRAPHY AND MASS SPECTROMETRY}

GC/MS analyses of all samples were performed using a mass spectrometer connected to a Shimadzu GCMSQP2010 plus gas chromatograph (Kyoto, Japan), equipped with a HP-5MS column (5\%-phenylmethylpolysiloxane, $30 \mathrm{~m} \times 0.25 \mathrm{~mm}$ id, $0.25 \mu \mathrm{m}$ film thickness). Helium gas was used as the carrier, at a flow rate of $1.0 \mathrm{~mL} / \mathrm{min}$. The injector temperature was $250^{\circ} \mathrm{C}$ and the oven temperature was initially set at $60{ }^{\circ} \mathrm{C}$ and increased at $3{ }^{\circ} \mathrm{C}$ per min, until reaching $260^{\circ} \mathrm{C}$, at which time this temperature was held for $40 \mathrm{~min}$.

The mass spectrometer was operated in full scan mode from 40 to $1000 \mathrm{~m} / \mathrm{z}$, at an interface temperature of $240{ }^{\circ} \mathrm{C}$, and the samples were ionized with an electron beam of $70 \mathrm{eV}$.

Identification of chemical constituents was performed by comparing the mass spectra with data from the NIST/ EPA/NIH Mass Spectral Library (Version 2.0).

The linear retention indices were calculated according to the Kovats method (KI), using a mixture of C6-C28 $\mathrm{n}$-alkanes as external references (Sigma-Aldrich, St. Louis, MO, USA).

Only compounds with retention times and fragmentation patterns that matched reference compounds or spectral data available in literature were considered reliable, and thus included in the text.

\section{HPLC-ESI-EM}

These analyses were carried out using a Bruker HPLC instrument (MicroTOFII) coupled to a diode-array detector and a mass spectrometer with electrospray ionization (ESI-MS). The mass spectrometer was operated in the positive ion mode and provided mass spectra data from $\mathrm{m} / \mathrm{z} 50$ to 1000

Twenty microliters of each sample $\left(5 \mathrm{mg} \mathrm{mL}^{-1}\right)$ were injected onto a Kinetex C18 column $(150 \mathrm{~mm} \times 3 \mathrm{~mm}, 2.6$ $\mu \mathrm{m}$; Phenomenex $\left.{ }^{\circledR}\right)$, at room temperature. For elution, a mixture of methanol/ammonium acetate $(1 \mathrm{mM})(70: 30)$ (Eluent A) and acetonitrile/ ammonium acetate (10 mM) in methanol (98:2) (Eluent B) were used, at a flow rate of $0.7 \mathrm{~mL} \mathrm{~min}^{-1}$. The gradient was as follows: $0 \% \mathrm{~B}$ (0 to 1 $\mathrm{min}$ ); 0 to $60 \% \mathrm{~B}$ (1-25 min); 60 to $100 \% \mathrm{~B}$ (25 to $27 \mathrm{~min}$ ); $100 \% \mathrm{~B}(27$ to $30 \mathrm{~min}) 100$ to $0 \% \mathrm{~B}(30-31 \mathrm{~min}) ; 0 \% \mathrm{~B}$ (31-32 min). Substances were identified with the aid of the computational deconvolution process.
THIN-LAYER CHROMATOGRAPHY/BIOAUTOGRAPHY FOR DETECTION OF ANTIFUNGAL ACTIVITY

The assay was conducted using the microorganism Cladosporium cladosporioides Fresen (SPC 140), which have been maintained at the Instituto de Botânica, São Paulo, Brazil. This fungus was cultured on potato dextrose agar (Difco) for 12 days until sporulation. The spore suspension was then extracted in a solution containing glucose and salt (Homans \& Fuchs 1970, Rahalison et al. 1994) to a final concentration of 108 spores $\mathrm{mL}^{-1}$ and used for qualitative assessment of the antifungal activity of the crude seaweed extract and fractions.

The test was carried out by applying $10 \mu \mathrm{L}$ of a solution containing $100 \mu \mathrm{g}$ of crude extract or fractions on silica gel GF 254 TLC plates (Merck, Germany) in DCM/MeOH (98.5:1.5 v/v), which were developed in tanks lined with solvent-saturated Whatman No. 3M. After thorough air drying, the chromatograms were sprayed with fungal spore suspensions and incubated for $72 \mathrm{~h}$ at $28{ }^{\circ} \mathrm{C}$. Antifungal compounds on the developed plates appeared as clear zones against the dark backgrounds of the TLC plates. Nystatin was used as positive controls.

\section{RESULTS}

In Figure 2A, the inhibitory activity exerted by a $D$. marginata hexane extract against the fungi Cladosporium cladosporioides is shown. Likewise, the Figure 2B displays the antifungal bioautographic assay of all fractions of the hexane extract and shows that all of them were active.

The combination of similar fractions from the C-I chromatographic process resulted in 12 fractions, five of which were analyzed by GC/MS. The refractionation (process C-II) of the pooled fractions (Fractions 4-9 from chromatographic process C-I) yielded 16 new fractions, 8 of which were analyzed by GC/MS.

The characteristic algal metabolites identified by the GC/MS analyses are listed in Table 1; among them, there are the diterpene Phytol, which is a product of Chlorophyll degradation (Olofsson et al. 2014) and Demosterol, a steroid commonly found in red alga (Sharanagat et al. 2019). 


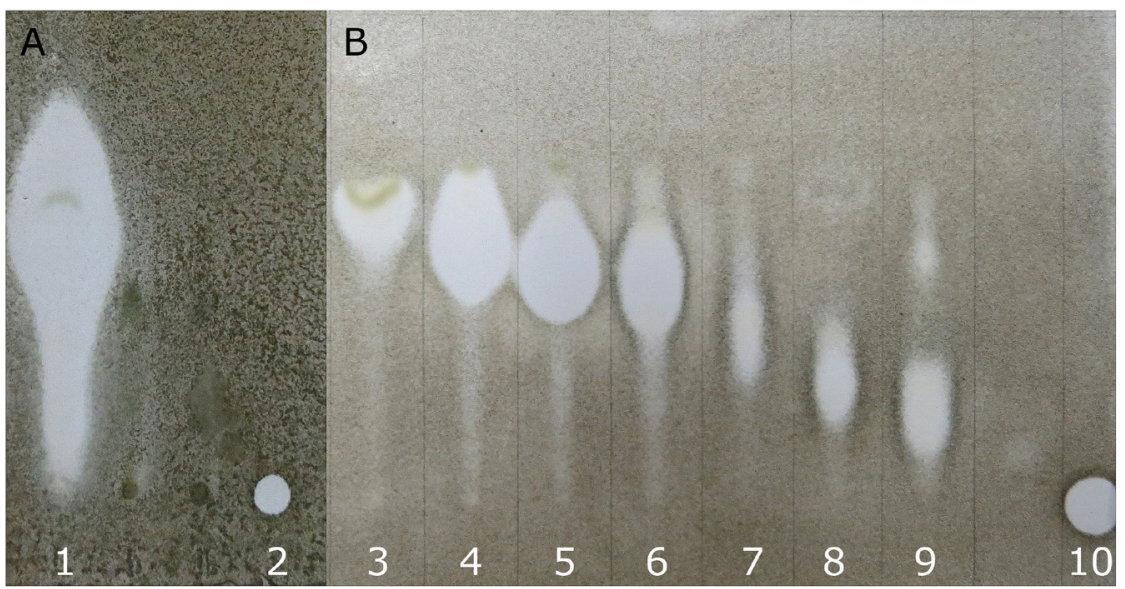

Figure 2. Antifungal TLC (thin layer chromatography) bioautographies of the D. marginata against Cladosporium cladosporioides. A) Hexane extract (1) and Positive control (nystatin)(2). B) Combined fractions from (C-1): 3, (4-9); 4, (10-15); 5, (16-27); 6, (28-33); 7, (34-36); 8, (37-38); 9, (39-40) and 10. Positive control (Nystatin). Mobile phases (A and B) dichloromethane/methanol 98.5:1.5 v/v/ Detección por bioautografías de compuestos antifúngicos frente al hongo Cladosporium cladosporioides encontrado en D. marginata. A) Extracto hexánico (1) y Control positivo (nistatina) (2). B) Fracciones combinadas de (C-I): 3, (4-9); 4, (10-15); 5, (16-27); 6, (28-33); 7, (34-36); 8, (37-38); 9, (39-40) y 10. Control positivo (nistatina). Fases móviles (A y B) diclorometano/metanol 98,5:1,5 v/v

Table 1. Substances synthesized by algae and identified in $D$. marginata by gas chromatography/mass spectrometry (GC/MS)/ Sustancias sintetizadas por algas e identificadas en $D$. marginata por cromatografía de gases/espectrometría de masas (GC/MS)

\begin{tabular}{|c|c|c|c|c|c|c|c|c|c|}
\hline $\mathrm{N}^{\circ}$ & $\begin{array}{l}\text { Material } \\
\text { analyzed }\end{array}$ & $\operatorname{Rt}(\min )$ & SI & KI & Substance & Other names & $\begin{array}{l}\text { Molecular } \\
\text { formula }\end{array}$ & $\begin{array}{c}\text { Molecular } \\
\text { mass } \\
\left(\mathrm{g} \mathrm{mol}^{-1}\right)\end{array}$ & Reference \\
\hline 1 & $\mathrm{~A} ; \mathrm{B} ; \mathrm{C}$ & 48.850 & 97 & 2114.6 & Phytol & $\begin{array}{l}\text { 3,7,11,15-tetramethyl-2- } \\
\text { hexadecen-1-ol }\end{array}$ & $\mathrm{C}_{20} \mathrm{H}_{40} \mathrm{O}$ & 296.539 & Souza \& Nes 1969 \\
\hline 2 & A & 54.200 & 96 & 2300.5 & Triacontane & $\mathrm{N}$-triacontane & $\mathrm{C}_{30} \mathrm{H}_{62}$ & 422.826 & Moustafa et al. 2008 \\
\hline 3 & $\mathrm{~A} ; \mathrm{C} ; \mathrm{D} ; \mathrm{E}$ & 78.117 & 91 & 3134.8 & Demosterol & Cholesta-5,24-dien-3-ol & $\mathrm{C}_{27} \mathrm{H}_{44} \mathrm{O}$ & 384.648 & Burn et al. 1957 \\
\hline 4 & C; D & 74.775 & 94 & 3057.5 & Cholesta-5, 22-dien-3- $\beta$-ol & 22-dehydrocholesterol & $\mathrm{C}_{27} \mathrm{H}_{44} \mathrm{O}$ & 384.648 & Burn et al. 1957 \\
\hline 5 & $\mathrm{~A} ; \mathrm{C} ; \mathrm{D}$ & 76.317 & 87 & 3097.5 & Epicholesterol & Cholest-5-en-3-ol & $\mathrm{C}_{27} \mathrm{H}_{46} \mathrm{O}$ & 386.664 & $\begin{array}{l}\text { Govindan et al. } 1993 \text {, } \\
\text { Plouguerné et al. } 2006\end{array}$ \\
\hline
\end{tabular}

Rt: Retention time, SI: Similarity index; KI: Kovats index

A: total hexane extract; B: C-I (Fr 10-15); C: C-I (Fr 16-27); D: C-I (Fr 28-33); E: C-I (Fr 34-37)

Substances that can be used to toiletries and personal care products, which were also herein identified, are in Table 2. They include myristic, oleic and palmitoleic acids, compounds produced by algae but that are also synthesized on an industrial scale to supply cosmetic companies (Gressler et al. 2010, Zielinska \& Nowak 2014).
The emerging pollutants detected by GC/MS are shown in Table 3. In total, 23 compounds were identified, with industrial contaminants being the most prevalent, followed by personal care products (Octinoxate). Pharmaceuticals (Pregnenolone methyl ether), household cleaning products (Cyclohexasiloxane), food additives (Ciclotene), endocrine disruptors (Nonylphenol) and plasticizers (Diisobutyl phthalate) were also identified. 
Table 2. Substances synthesized by algae and produced for various industrial uses, identified in $D$. marginata by gas chromatography/mass spectrometry (GC/MS) / Sustancias sintetizadas por algas y producidas para varios usos industriales, identificadas en $D$. marginata por cromatografía de gases/espectrometría de masas (GC/MS)

\begin{tabular}{|c|c|c|c|c|c|c|c|c|c|c|}
\hline $\mathrm{N}^{\circ}$ & $\begin{array}{l}\text { Material } \\
\text { analyzed }\end{array}$ & Rt (min) & SI & KI & Substance & Other names & $\begin{array}{l}\text { Molecular } \\
\text { formula }\end{array}$ & $\begin{array}{l}\text { Molecular } \\
\text { mass }(\mathrm{g} \\
\left.\text { mol }^{-1}\right)\end{array}$ & $\begin{array}{l}\text { Substance } \\
\text { information }\end{array}$ & Reference/ Patent \\
\hline 1 & A & 15.183 & 85 & 1200.1 & Dodecane & Dihexyl & $\mathrm{C}_{12} \mathrm{H}_{26}$ & 170.340 & I; II & EFSA 2008, EP0002349 B1 \\
\hline 2 & A & 23.650 & 92 & 1400.9 & Tetradecane & $\mathrm{N}$-tetradecane & $\mathrm{C}_{14} \mathrm{H}_{30}$ & 198.394 & II; VIII & $\begin{array}{l}\text { Fujimura et al. 1990, Kalhor } \\
\text { et al. 2017, EP0002349 B1 }\end{array}$ \\
\hline 3 & $\mathrm{~A} ; \mathrm{B} ; \mathrm{C} ; \mathrm{G} ; \mathrm{H}$ & 28.817 & 93 & 1529.6 & Dihydroactinidiolide & $\begin{array}{l}\text { 5,6,7,7a-tetrahydro- } \\
\text { 4,4,7a-trimethyl- } \\
\text { 2(4H)-benzofuranone }\end{array}$ & $\mathrm{C}_{11} \mathrm{H}_{16} \mathrm{O}_{2}$ & 180.247 & $\mathrm{~V}$ & $\begin{array}{l}\text { Yao et al. 1998, Xian et al. } \\
\text { 2006, Borik 2014, } \\
\text { US20130014771 A1 }\end{array}$ \\
\hline 4 & $\mathrm{~A} ; \mathrm{C} ; \mathrm{G} ; \mathrm{H}$ & 31.583 & 97 & 1601.0 & N-hexadecane & Cetane & $\mathrm{C}_{16} \mathrm{H}_{34}$ & 226.448 & I & Islam et al. 2013 \\
\hline 5 & $\mathrm{~A} ; \mathrm{G}$ & 35.033 & 97 & 1694.4 & Heptadecene & 1-heptadecene & $\mathrm{C}_{17} \mathrm{H}_{34}$ & 238.459 & I; V; VIII & $\begin{array}{l}\text { Rezanka et al. } 1982 \text {, } \\
\text { Yamamoto et al. } 2014 \text {, } \\
\text { US20150376544 A1 }\end{array}$ \\
\hline 6 & $\mathrm{~A} ; \mathrm{G} ; \mathrm{H}$ & 35.433 & 97 & 1705.5 & Heptadecane & N-heptadecane & $\mathrm{C}_{17} \mathrm{H}_{36}$ & 240.475 & $\mathrm{~V}$ & $\begin{array}{l}\text { McInnes et al. } 1979 \text {, } \\
\text { Sugisawa et al. 1990, } \\
\text { Oliveira et al. 2012, } \\
\text { US8933334 B2 }\end{array}$ \\
\hline 7 & $\mathrm{C}$ & 35.808 & 94 & 1716.1 & Tetradecene & 1-tetradecene & $\mathrm{C}_{14} \mathrm{H}_{28}$ & 196.378 & $\mathrm{I} ; \mathrm{V}$ & Yamamoto et al. 2014 \\
\hline 8 & A & 37.575 & 94 & 1766.2 & Myristic acid & Tetradecanoic acid & $\mathrm{C}_{14} \mathrm{H}_{28} \mathrm{O}_{2}$ & 228.376 & I; II; V & $\begin{array}{l}\text { Widianingsih et al. 2012, } \\
\text { Zielińska A \& I Nowak } \\
\text { 2014, El-Maghraby \& } \\
\text { Fakhry } 2015\end{array}$ \\
\hline 9 & A; G; H & 38.783 & 96 & 1800.5 & Octadecane & N-octadecane & $\mathrm{C}_{18} \mathrm{H}_{38}$ & 254.502 & I & $\begin{array}{l}\text { Borik 2014, EP0002349 B1, } \\
\text { US8961622 B2 }\end{array}$ \\
\hline 10 & A & 39.675 & 93 & 1827.0 & Pentadecanoic acid & Pentadecylic acid & $\mathrm{C}_{15} \mathrm{H}_{30} \mathrm{O}_{2}$ & 242.403 & I & $\begin{array}{l}\text { Plaza et al. 2010, } \\
\text { EP0003032 B1, EP0022871 } \\
\text { B1 }\end{array}$ \\
\hline 11 & A; B; C; G; H & 40.317 & 94 & 1846.1 & Phytone & $\begin{array}{l}\text { 6,10,14-trimethyl-2- } \\
\text { pentadecanone }\end{array}$ & $\mathrm{C}_{18} \mathrm{H}_{36} \mathrm{O}$ & 268.485 & I & $\begin{array}{l}\text { Eltz et al. 2010, Adegoke et } \\
\text { al. 2015, Hatem et al. } 2016\end{array}$ \\
\hline 12 & $\mathrm{~A} ; \mathrm{H}$ & 42.142 & 95 & 1900.5 & Nonadecane & N-nonadecane & $\mathrm{C}_{19} \mathrm{H}_{40}$ & 268.529 & $\mathrm{~V}$ & $\begin{array}{l}\text { Abou-El-Wafa et al.2011, } \\
\text { US8859471 B2 }\end{array}$ \\
\hline 13 & $\mathrm{G} ; \mathrm{H}$ & 42.983 & 92 & 1926.7 & Methyl palmitate & $\begin{array}{l}\text { Hexadecanoic acid, } \\
\text { methyl ester }\end{array}$ & $\mathrm{C}_{17} \mathrm{H}_{34} \mathrm{O}_{2}$ & 270.457 & $\begin{array}{l}\text { I; III; IV; } \\
\text { V; VI }\end{array}$ & $\begin{array}{l}\text { Knaggs et al. 1965, Parris et } \\
\text { al. 1973, Lee et al. 2010, El- } \\
\text { Demerdash 2011, } \\
\text { US20160089464 B1, } \\
\text { US20160089317 B1 }\end{array}$ \\
\hline 14 & $\mathrm{~A} ; \mathrm{F} ; \mathrm{G} ; \mathrm{H}$ & 43.808 & 95 & 1952.3 & Palmitoleic acid & 9-hexadecenoic acid & $\mathrm{C}_{16} \mathrm{H}_{30} \mathrm{O}_{2}$ & 254.414 & V & Pereira et al. 2012 \\
\hline 15 & $\mathrm{~A} ; \mathrm{F} ; \mathrm{G} ; \mathrm{H}$ & 44.300 & 92 & 1967.7 & Palmitic acid & N-hexadecanoic acid & $\mathrm{C}_{16} \mathrm{H}_{32} \mathrm{O}_{2}$ & 256.430 & $\mathrm{~V}$ & $\begin{array}{l}\text { Kamenarska et al. 2006, } \\
\text { Pereira et al. 2012, Sahu et } \\
\text { al. 2013, US20150191607 } \\
\text { A1, US4537782 A }\end{array}$ \\
\hline 16 & $\mathrm{G} ; \mathrm{H}$ & 45.175 & 95 & 1994.9 & Ethyl palmitate & Ethyl hexadecanoate & $\mathrm{C}_{18} \mathrm{H}_{36} \mathrm{O}_{2}$ & 284.484 & $\mathrm{~V}$ & $\begin{array}{l}\text { Cortés et al. 2014, } \\
\text { US20160089311 A1, } \\
\text { US20160089317 A1 }\end{array}$ \\
\hline 17 & A; G; H & 45.367 & 96 & 2000.9 & Eicosane & Icosane & $\mathrm{C}_{20} \mathrm{H}_{42}$ & 282.556 & I & $\begin{array}{l}\text { Karabay-Yavasoglu et al. } \\
\text { 2007, US8859471 B2 }\end{array}$ \\
\hline 18 & $\mathrm{G} ; \mathrm{H}$ & 48.158 & 81 & 2091.5 & Propyl palmitate & $\begin{array}{l}\text { Propyl } \\
\text { hexadecanoate }\end{array}$ & $\mathrm{C}_{19} \mathrm{H}_{38} \mathrm{O}_{2}$ & 298.511 & $\mathrm{~V}$ & $\begin{array}{l}\text { Lui et al. 2008, } \\
\text { US20160051455 A1 }\end{array}$ \\
\hline 19 & $\mathrm{~A} ; \mathrm{G}$ & 48.425 & 94 & 2100.2 & Heneicosane & Methyl-eicosane & $\mathrm{C}_{21} \mathrm{H}_{44}$ & 296.583 & I & $\begin{array}{l}\text { Abou-El-Wafa et al. 2011, } \\
\text { US8598098 B2 }\end{array}$ \\
\hline 20 & $\mathrm{~A} ; \mathrm{G} ; \mathrm{H}$ & 50.021 & 89 & 2154.4 & Neophyitadiene & $\begin{array}{l}\text { 2-(4,8,12- } \\
\text { trimethyltrydecyl) } \\
\text { buta -1,3-diene }\end{array}$ & $\mathrm{C}_{20} \mathrm{H}_{38}$ & 278.524 & $\mathrm{~V}$ & $\begin{array}{l}\text { Plaza et al. 2010, Oliveira et } \\
\text { al. 2012, Peres et al. 2012, } \\
\text { US2012211015 A1 }\end{array}$ \\
\hline 21 & $\mathrm{G} ; \mathrm{H}$ & 50.417 & 86 & 2167.9 & Ethyl oleate & Oleic acid ethyl ester & $\mathrm{C}_{20} \mathrm{H}_{38} \mathrm{O}_{2}$ & 310.522 & $\begin{array}{l}\text { II; III; V; } \\
\text { VI }\end{array}$ & $\begin{array}{l}\text { Bookstaff et al. } 2003 \text {, } \\
\text { Phaechamud \& Savedkairop } \\
\text { 2012, Castillo } \text { et al. } 2012 \text {, } \\
\text { Abdel-Aal } \text { et al. } 2015\end{array}$ \\
\hline 22 & $\mathrm{~A} ; \mathrm{G} ; \mathrm{H}$ & 51.383 & 95 & 2200.8 & Docosane & N-docosane & $\mathrm{C}_{22} \mathrm{H}_{46}$ & 310.610 & II & $\begin{array}{l}\text { Karabay-Yavasoglu et al. } \\
2007 \text {, US8980346 B2 }\end{array}$ \\
\hline 23 & $\mathrm{~A} ; \mathrm{F} ; \mathrm{G} ; \mathrm{H}$ & 59.967 & 89 & 2518.1 & Oleic acid & $\begin{array}{l}\text { Cis-9-octadecenoic } \\
\text { acid }\end{array}$ & $\mathrm{C}_{18} \mathrm{H}_{34} \mathrm{O}_{2}$ & 282.468 & I; V; VI & $\begin{array}{l}\text { Khotimchenko et al. } 2002 \text {, } \\
\text { Huang et al. 2010, Sahu et } \\
\text { al. } 2013\end{array}$ \\
\hline
\end{tabular}

Rt: Retention time, SI: Similarity index; KI: Kovats index; A: total hexane extract; B: C-I (Fr 10-15); C: C-I (Fr 16-27); F: C-I (Fr 38); G: C-II (Fr 26-29);

H: C-II (Fr30-33).

I: industrial inputs; II: food additives, III: drugs; IV: household products; V: products for personal use; VI: plasticizers; VIII: flame retardants 
Table 3. Emergent pollutants identified in D. marginata by gas chromatography/mass spectrometry (GC/MS) / Contaminantes emergentes identificados en $D$. marginata por cromatografía de gases/espectrometría de masas (GC/MS)

\begin{tabular}{|c|c|c|c|c|c|c|c|c|c|c|}
\hline $\mathrm{N}^{0}$ & $\begin{array}{l}\text { Material } \\
\text { analyzed }\end{array}$ & $\begin{array}{c}\mathrm{Rt} \\
(\mathrm{min})\end{array}$ & SI & KI & Substance & Other name & $\begin{array}{l}\text { Molecular } \\
\text { formula }\end{array}$ & $\begin{array}{l}\text { Molecular } \\
\text { mass } \\
\left(\mathrm{g} \mathrm{mol}^{-1}\right)\end{array}$ & $\begin{array}{l}\text { Substance } \\
\text { information }\end{array}$ & Reference/ Patent \\
\hline 1 & $\mathrm{C} ; \mathrm{D} ; \mathrm{E} ; \mathrm{H}$ & 5.267 & 97 & 913.8 & Tetrachloroethane & 1,1,2,2-tetrachloroethane & $\mathrm{C}_{2} \mathrm{H}_{2} \mathrm{Cl}_{4}$ & 167.838 & I & ATSDR 2008 \\
\hline 2 & A & 10.933 & 73 & 1095.3 & $\begin{array}{l}\text { 2-hydroxy-3-methyl-2- } \\
\text { cyclopenten-1-one }\end{array}$ & Ciclotene & $\mathrm{C}_{6} \mathrm{H}_{8} \mathrm{O}_{2}$ & 112.128 & II & $\begin{array}{l}\text { ЕР0080600 B1, } \\
\text { EP0413368 B1 }\end{array}$ \\
\hline 3 & A & 16.175 & 76 & 1223.5 & Cyclohexylpiperidine & 1-piperidinocyclohexane & $\mathrm{C}_{11} \mathrm{H}_{21} \mathrm{~N}$ & 167.296 & III & $\begin{array}{l}\text { EP0392317 B1, } \\
\text { EP0406111 B1 }\end{array}$ \\
\hline 4 & A & 19.442 & 95 & 1300.5 & Tridecane & N-tridecane & $\mathrm{C}_{13} \mathrm{H}_{28}$ & 184.367 & I & EP0002002 B1 \\
\hline 5 & $\begin{array}{l}\mathrm{A} ; \mathrm{C} ; \mathrm{D} ; \mathrm{E} \\
\mathrm{F} ; \mathrm{H}\end{array}$ & 20.825 & 90 & 1333.5 & $\begin{array}{l}\text { Dodecamethylcyclohexasil } \\
\text { oxane }\end{array}$ & Cyclohexasiloxane & $\begin{array}{c}\mathrm{C}_{12} \mathrm{H}_{36} \mathrm{O}_{6} \mathrm{Si} \\
6\end{array}$ & 444.924 & IV; V & $\begin{array}{l}\text { Horii \& Kannan 2008, } \\
\text { EP0285364 B1, } \\
\text { EP0246007 }\end{array}$ \\
\hline 6 & $\mathrm{~A} ; \mathrm{H}$ & 24.792 & 93 & 1429.1 & $\alpha$-ionone & Iraldeine & $\mathrm{C}_{13} \mathrm{H}_{20} \mathrm{O}$ & 192.302 & $\mathrm{~V}$ & $\begin{array}{l}\text { Adams et al. } 1996 \text {, } \\
\text { Lalko et al. } 2007 \text {, } \\
\text { Brechbill 2009, 2012; } \\
\text { CA2813334 A1 }\end{array}$ \\
\hline 7 & $\mathrm{~A} ; \mathrm{B}$ & 27.150 & 88 & 1487.2 & $\beta$-ionone & $\begin{array}{l}\text { 4-(2,6,6-trimethyl-1- } \\
\text { cyclohexen-1-yl)-3- } \\
\text { buten-2-one }\end{array}$ & $\mathrm{C}_{13} \mathrm{H}_{20} \mathrm{O}$ & 192.302 & II; I & $\begin{array}{l}\text { Lalko et al. } 2007 \text {, } \\
\text { EP0012246 B1 }\end{array}$ \\
\hline 8 & $\mathrm{~A} ; \mathrm{G} ; \mathrm{H}$ & 27.708 & 95 & 1501.0 & Pentadecane & N-pentadecane & $\mathrm{C}_{15} \mathrm{H}_{32}$ & 212.421 & I & $\begin{array}{l}\text { El-Boujaddaini et al. } \\
\text { 2010, EP0000819 B1 }\end{array}$ \\
\hline 9 & $\mathrm{C}$ & 33.840 & 94 & 1662.1 & 1-octadecanol & Stearyl alcohol & $\mathrm{C}_{18} \mathrm{H}_{38} \mathrm{O}$ & 270.501 & I; V; VI & $\begin{array}{l}\text { Bingham \& Cohrssen } \\
\text { 2012, Shore \& Shelley } \\
2015 \text {, US20160089321 } \\
\text { A1, US20160089323 } \\
\text { A1 }\end{array}$ \\
\hline 10 & $\mathrm{~F}$ & 35.108 & 89 & 1696.4 & Nonylphenol & N-nonylphenol & $\mathrm{C}_{15} \mathrm{H}_{24} \mathrm{O}$ & 220.356 & IV; VII & $\begin{array}{l}\text { Duan et al. 2016, He et } \\
\text { al. 2016, Sieppi et al. } \\
\text { 2016, EP0004108 B1 }\end{array}$ \\
\hline 11 & $\mathrm{G} ; \mathrm{H}$ & 37.139 & 92 & 1753.9 & Octinoxate & $\begin{array}{l}\text { 2-ethylhexyl 4- } \\
\text { methoxycinnamate }\end{array}$ & $\mathrm{C}_{18} \mathrm{H}_{26} \mathrm{O}_{3}$ & 290.403 & $\mathrm{~V}$ & $\begin{array}{l}\text { Schlumpf et al. 2004, } \\
\text { Axelstad et al. 2011, } \\
\text { US20160089317 A1 }\end{array}$ \\
\hline 12 & $\begin{array}{l}\mathrm{A} ; \mathrm{B} ; \mathrm{C} ; \mathrm{D} ; \\
\mathrm{G} ; \mathrm{H}\end{array}$ & 41.092 & 97 & 1869.2 & Diisobutyl phthalate & $\begin{array}{l}\text { 1,2-benzenedicarboxylic } \\
\text { acid, bis(2- } \\
\text { methylpropyl) ester }\end{array}$ & $\mathrm{C}_{16} \mathrm{H}_{22} \mathrm{O}_{4}$ & 278.348 & VI & $\begin{array}{l}\text { Poon et al. 1997, Koch } \\
\text { et al. 2006, Gao \& Wen } \\
2016\end{array}$ \\
\hline 13 & $\mathrm{C}$ & 41.433 & 96 & 1879.4 & N-hexadecanol-1-ol & Cetilic alcohol & $\mathrm{C}_{16} \mathrm{H}_{34} \mathrm{O}$ & 242.447 & II; III; V & US8865144 A1 \\
\hline 14 & $\mathrm{G} ; \mathrm{H}$ & 41.617 & 86 & 1884.8 & Homomenthyl salicylate & $\begin{array}{l}3,3,5- \\
\text { trimethylcyclohexyl } \\
\text { salicylate }\end{array}$ & $\mathrm{C}_{16} \mathrm{H}_{22} \mathrm{O}_{3}$ & 262.349 & V & $\begin{array}{l}\text { Rietschel \& Lewis } \\
\text { 1978, Sambandan \& } \\
\text { Ratner 2011, Jiménez- } \\
\text { Díaz et al. } 2013\end{array}$ \\
\hline 15 & A & 44.150 & 91 & 1963.0 & Methyl glycol phthalate & $\begin{array}{l}\text { 1,2-benzenedicarboxylic } \\
\text { acid, bis ( } 2 \text { - } \\
\text { methoxyethyl) ester }\end{array}$ & $\mathrm{C}_{14} \mathrm{H}_{18} \mathrm{O}_{6}$ & 282.292 & I; VII & $\begin{array}{l}\text { Poon et al. 1997, Koch } \\
\text { et al. 2006, Gao \& Wen } \\
2016\end{array}$ \\
\hline 16 & $\mathrm{~A}$ & 51.033 & 93 & 2188.8 & Butyl hexadecanoate & $\begin{array}{l}\text { Hexadecanoic acid, } \\
\text { butyl ester }\end{array}$ & $\mathrm{C}_{20} \mathrm{H}_{40} \mathrm{O}_{2}$ & 312.538 & II; V & Khan et al. 2016 \\
\hline 17 & B; G; H & 56.883 & 95 & 2399.3 & Diethylhexyl adipate & $\begin{array}{l}\text { Hexanedioic acid, bis(2- } \\
\text { ethylhexyl) ester }\end{array}$ & $\mathrm{C}_{22} \mathrm{H}_{42} \mathrm{O}_{4}$ & 370.574 & I; V; VI & $\begin{array}{l}\text { Jobling et al. } 1995 \text {, } \\
\text { Fasano et al. } 2012 \text {, } \\
\text { US20160089321 A1 }\end{array}$ \\
\hline 18 & $\mathrm{G} ; \mathrm{H} ; \mathrm{I}$ & 60.717 & 95 & 2548.0 & Bis(2-ethylhexyl)phthalate & $\begin{array}{l}\text { 1,2-benzenedicarboxylic } \\
\text { acid, 1,2-bis( } 2 \text { - } \\
\text { ethylhexyl) ester }\end{array}$ & $\mathrm{C}_{24} \mathrm{H}_{38} \mathrm{O}_{4}$ & 390.564 & I; VII & $\begin{array}{l}\text { Poon et al. 1997, Koch } \\
\text { et al. 2006, Gao \& Wen } \\
\text { 2016, US20160075671 } \\
\text { A1 }\end{array}$ \\
\hline 19 & $\begin{array}{l}\mathrm{A} ; \mathrm{C} ; \mathrm{D} ; \mathrm{F} ; \\
\mathrm{G}\end{array}$ & 60.775 & 97 & 2550.3 & Monoetylhexyl phthalate & $\begin{array}{l}\text { 1,2-benzenedicarboxylic } \\
\text { acid, mono( } 2 \text { - } \\
\text { ethylhexyl) ester }\end{array}$ & $\mathrm{C}_{16} \mathrm{H}_{22} \mathrm{O}_{4}$ & 278.344 & $\mathrm{I} ; \mathrm{VI}$ & $\begin{array}{l}\text { Koch et al. 2006, Poon } \\
\text { et al. 1997, Gao \& Wen } \\
2016\end{array}$ \\
\hline 20 & $\mathrm{~A} ; \mathrm{D}$ & 64.458 & 95 & 2699.9 & Tetracontane & $\mathrm{N}$-tetracontane & $\mathrm{C}_{40} \mathrm{H}_{82}$ & 563.096 & I; III & $\begin{array}{l}\text { Mortimer \& Luke 1966, } \\
\text { Vazquez \& Mansoori } \\
\text { 2000, US8013091 B2 }\end{array}$ \\
\hline 21 & $\mathrm{C} ; \mathrm{D}$ & 64.650 & 75 & 2708.1 & Pregnenolone methyl ether & $\begin{array}{l}\text { Pregnenolone 3-methyl } \\
\text { ether }\end{array}$ & $\mathrm{C}_{22} \mathrm{H}_{34} \mathrm{O}_{2}$ & 330.512 & III & Marx et al. 2011 \\
\hline 22 & $\mathrm{C} ; \mathrm{D} ; \mathrm{E} ; \mathrm{G}$ & 66.267 & 91 & 2777.0 & Erucylamide & 13-docosenamide & $\mathrm{C}_{22} \mathrm{H}_{43} \mathrm{NO}$ & 337.592 & I & $\begin{array}{l}\text { US20160083586 A1, } \\
\text { US20130177703 A1 }\end{array}$ \\
\hline
\end{tabular}

Rt: Retention time, SI: Similarity index; KI: Kovats index; A: total hexane extract; B: C-I (Fr 10-15); C: C-I (Fr 16-27); D: C-I (Fr 28-33); E: C-I (Fr 34-37);

F: C-I (Fr 38); G: C-II (Fr 26-29), H: C-II (Fr 30-33) e I: C-II (Fr 34-38).

I: industrial inputs; II: food additives, III: drugs; IV: household products; V: products for personal use; VI: plasticizers; VII: endocrine disruptors 
The sixteen pollutants which were identified by HPLC/ MS technique compounds are gathered in Table 4. Seven of these compounds are used for personal care, four as food additives, and two are pharmaceuticals. In fact, the drugs dipyrone and ethinylestradiol were only detected by HPLC/MS.
Figure 3 shows the structural formulas of some of these pollutants (Kim et al. 2019).

Table 4. Substances identified in D. marginata by liquid chromatography/mass spectrometry (HPLC/MS) / Sustancias identificadas en $D$. marginata por cromatografía de líquidos/espectrometria de masas (HPLC/MS)

\begin{tabular}{|c|c|c|c|c|c|c|}
\hline \multirow{2}{*}{$\mathrm{N}^{\circ}$} & \multirow{2}{*}{ Substance } & \multirow{2}{*}{$\begin{array}{l}\text { Molecular } \\
\text { formula }\end{array}$} & \multirow{2}{*}{$\begin{array}{l}\text { Molecular } \\
\text { mass } \\
\left(\mathrm{g} \mathrm{mol}^{-1}\right)\end{array}$} & \multicolumn{2}{|c|}{$[\mathrm{M}+\mathrm{H}]^{+}$} & \multirow{2}{*}{$\begin{array}{l}\text { Substance } \\
\text { information }\end{array}$} \\
\hline & & & & $\begin{array}{c}\text { Theoretical } \\
\mathrm{m} / \mathrm{z}\end{array}$ & $\begin{array}{c}\text { Observed } \\
\mathrm{m} / \mathrm{z}\end{array}$ & \\
\hline 1 & 3-methylcyclopenta-2-enone & $\mathrm{C}_{6} \mathrm{H}_{8} \mathrm{O}$ & 96.0575 & 97.0647 & 97.0644 & I; II; V \\
\hline 2 & $\alpha$-ionone & $\mathrm{C}_{13} \mathrm{H}_{20} \mathrm{O}$ & 192.302 & 193.1587 & 193.1562 & $\mathrm{~V}$ \\
\hline 3 & Dihydroactinidiolide & $\mathrm{C}_{11} \mathrm{H}_{16} \mathrm{O}_{2}$ & 180.247 & 181.1223 & 181.1228 & $\mathrm{~V}$ \\
\hline 4 & Diisobutyl Phthalate & $\mathrm{C}_{18} \mathrm{H}_{36} \mathrm{O}$ & 278.348 & 279.1590 & 279.1590 & VI \\
\hline 5 & n-butyl-9-octadecenamide & $\mathrm{C}_{22} \mathrm{H}_{43} \mathrm{NO}$ & 337.592 & 338.3417 & 338.3416 & I \\
\hline 6 & Palmitoleic acid & $\mathrm{C}_{16} \mathrm{H}_{30} \mathrm{O}_{2}$ & 254.414 & 255.2240 & 255.2276 & $\mathrm{~V}$ \\
\hline 7 & Homomenthyl salicylate & $\mathrm{C}_{16} \mathrm{H}_{22} \mathrm{O}_{3}$ & 262.349 & 263.1641 & 263.1645 & V \\
\hline 8 & Oxybenzone & $\mathrm{C}_{14} \mathrm{H}_{12} \mathrm{O}_{3}$ & 228.247 & 229.0859 & 229.0844 & $\mathrm{~V}$ \\
\hline 9 & 2-propenoic acid 3- (4-methoxyphenyl) -2-ethylhexyl ester (octinoxate) & $\mathrm{C}_{18} \mathrm{H}_{26} \mathrm{O}_{3}$ & 290.403 & 291.1954 & 291.1954 & $\mathrm{~V}$ \\
\hline 10 & Bis(2-ethylhexyl) phthalate & $\mathrm{C}_{24} \mathrm{H}_{38} \mathrm{O}_{4}$ & 390.564 & 391.2842 & 391.2853 & VI \\
\hline 11 & $\beta$-ionone & $\mathrm{C}_{13} \mathrm{H}_{20} \mathrm{O}$ & 192.302 & 193.1586 & 193.1555 & $\mathrm{I} ; \mathrm{II}$ \\
\hline 12 & 7,9-di-tert-butyl-1-oxaspiro $(4,5)$ deca-6-9-diene-1,8-dione & $\mathrm{C}_{17} \mathrm{H}_{24} \mathrm{O}_{3}$ & 276.376 & 277.1798 & 277.1787 & I \\
\hline 13 & Tributyl acetyl citrate & $\mathrm{C}_{20} \mathrm{H}_{34} \mathrm{O}_{8}$ & 402.484 & 403.2326 & 403.2344 & VI \\
\hline 15 & Dipyrone & $\mathrm{C}_{13} \mathrm{H}_{16} \mathrm{~N}_{3} \mathrm{NaO}_{4} \mathrm{~S}$ & 333.076 & 334.0831 & 334.0884 & III \\
\hline 16 & Ethinylestradiol & $\mathrm{C}_{20} \mathrm{H}_{24} \mathrm{O}_{2}$ & 296.403 & 297.1849 & 297.1832 & III \\
\hline
\end{tabular}

I: industrial inputs; II: food additives, III: drugs; V: products for personal use; VI: plasticizers

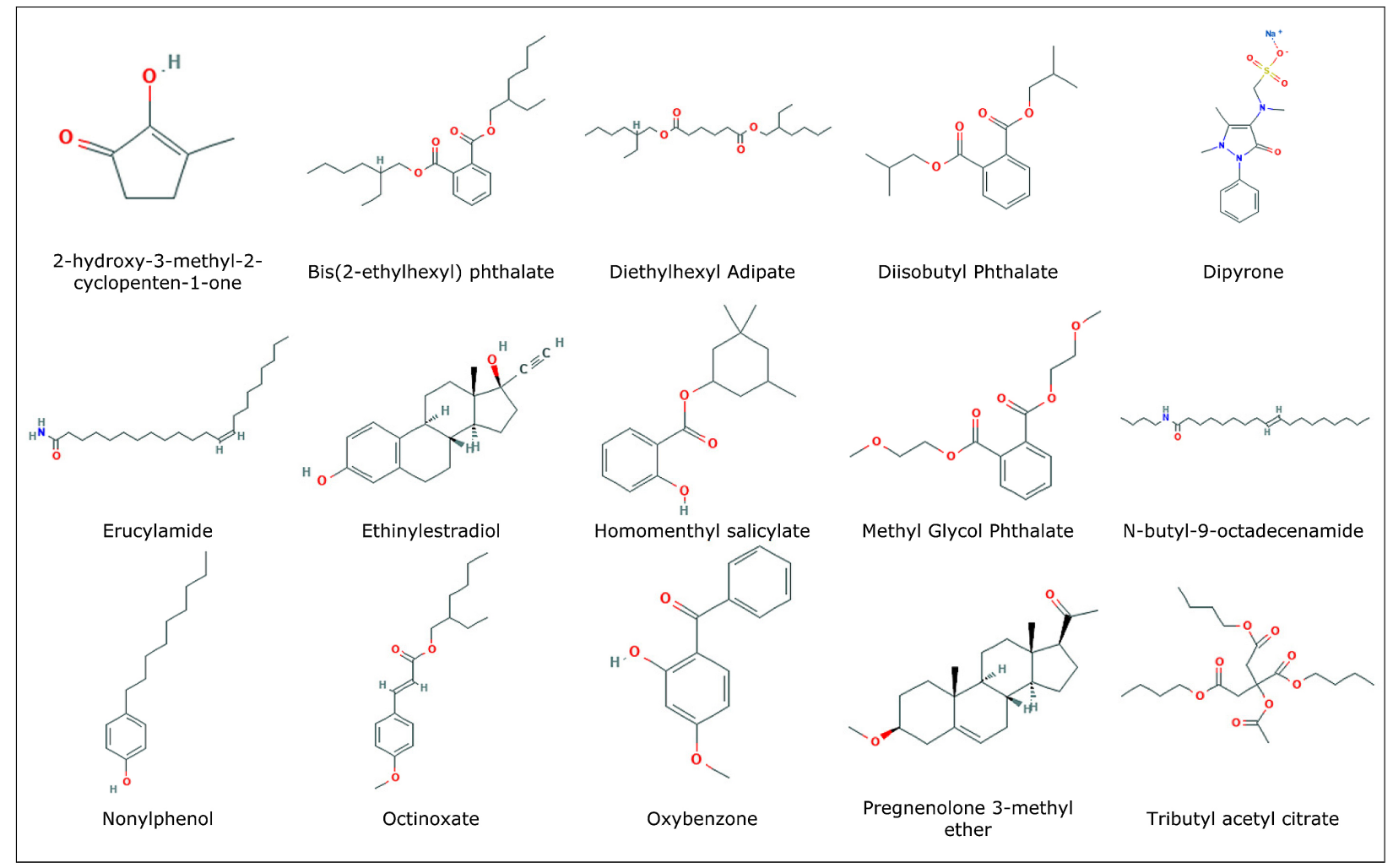

Figure 3. Chemical structure of some emergent pollutants identified in D. marginata (figure modified from Kim et al. 2019) / Estructura química de algunos contaminantes emergentes identificados en D. marginata (figura modificada de Kim et al. 2019) 


\section{Discussion}

Hexane extracts of $D$. marginata presented, in addition to its typical components, compounds with different origins and potential applications, indicating that the species is able to retain them in its thallus.

The main components of the hexane extracts are usually compounds of low molecular weight and low polarity, such as some hydrocarbons, fatty acids, terpenes and aromatics compounds. Antibiotic activities of any kind which were assigned to these extracts (essential oils) oftentimes are due to terpenes and aromatic compounds (Nazzaro et al. 2017).

Among the primary metabolites herein identified (Table 1) only one, eicosane, has antifungal activity (Ahsan et al. 2017).

Special mention should be made of fatty acids $\mathrm{C}_{12}, \mathrm{C}_{14}$ and $\mathrm{C}_{16}$ (Table 2), since they are valuable components of the human diet, precursors of the eicosanoid biosynthesis and bioregulators of several cellular processes (Gressler $e t$ al. 2010). Additionally, these compounds are considered to be a useful tool in chemotaxonomy, since they help to distinguish classes, families and genera of macroalgae (Khotimchenko 2005).

Among the compounds listed in Table 3, which are all pollutants, phthalates deserve special attention since they are very often isolated from macroalgae and sometimes mistakenly regarded as the natural components of these organisms (Chan et al. 2004, Gressler et al. 2012, Avio et al. 2016). Phathalates are not only antifungal compounds but also they may have toxic effects on humans and animals (Rowdhwal \& Chen 2018). Still in Table 3, various other substances which have adverse effects on living beings are listed (references cited in Table 3).

Apart from four variants of phthalates, the pollutants nonyphenol (Karley et al. 1997), and cyclohexasiloxane (Moustafa et al. 2013) have antifungal activity as well. The fungitoxic action of these compounds could justify much of the observed fungal inhibition, since only one algal metabolite showed this activity.

The adsorption of these pollutants relied on the ability of the structural polysaccharides of the macroalgae to sequester and accumulate substances, with which these compounds can electrostatically bond.

On the other hand, the molecules of these substances must have oxygen atoms (hydrogen receptors) which are the ones that make up carbonyl (from acids, esters and ketones) and phenolic hydroxyl groups as well, nitrogen atoms which make up amines and amides and sulfur atoms, for sulfate groups (Paoloni et al. 1975, Hay et al. 2004, Schaeffer 2008, Ouellette \& Rawn 2015).
The molecules of the sixteen pollutants herein identified have groups which can act as hydrogen receptors, by bonding with the numerous hydroxyl groups of sugar polymers, forming hydrogen bonds. The pollutants octinoxate, diisobutyl phthalate, homomenthyl salicylate, methyl glycol phthalate, pregnenolone 3-methyl ether, erucylamide, n-butyl-9-octadecenamide, oxybenzone, tributyl acetyl citrate, dipyrone, and 2-hydroxy-3-methyl-2cyclopenten-1-one have a caboxyl group. Ethinylestradiol, oxybenzone, nonylphenol, and homomenthyl salicylate are phenolic substances; erucylamide and n-butyl-9octadecenamide are amides and dipyrone is a sulfated substance (Fig. 3).

While the GC/MS method reliably identified emerging pollutants desorbed from the thallus of $D$. marginata, the HPLC/MS procedure, assisted by spectral deconvolution, proved to be a valuable tool for identifying non-metallic, polar, non-volatile and thermally unstable environmental contaminants. These types of compounds are often present in minute quantities in samples with a profuse mixture of compounds (Magi \& Di Carro 2016, Martín et al. 2017).

In conclusion, the significant number of pollutants detected by both chromatographic methods (GC and HPLC) indicate that macroalgae have the ability to retain a variety of substances dissolved or suspended in the aquatic environment. Due to the fact that some of the identified compounds were from pharmaceuticals residues and industrial debris, the present study has demonstrated that the red alga Dichotomaria marginata could be a reliable bioindicator for water pollution. Early detection and subsequent remediation will minimize the harmful effects associated with these pollutants, and benefit the human population, in general.

\section{ACKNOWLEDGEMENTS}

This study was financially supported by the Coordenação de Aperfeiçoamento de Pessoal de Nível Superior (CAPES, Brazil) - CAPES/AUXPE-CIMAR 1991/2014/Process $n^{\circ}$ 23038.001431/2014-75 and Fundação de Amparo à Pesquisa do Estado de São Paulo (FAPESP/ Process $n^{\circ}$ 2017/07346-0) (EMS).

\section{LITERATURE CITED}

Abdel-Aal EI, AM Haroon \& J Mofeed. 2015. Successive solvent extraction and GC-MS analysis for the evaluation of the phytochemical constituents of the filamentous green alga Spirogyra longata. Egyptian Journal of Aquatic Research 41(3): 233-246.

Abou-El-Wafa GS, KA Shaaban, MEE El-Naggar \& M Shaaban. 2011. Bioactive constituents and biochemical composition of the Egyptian brown alga Sargassum subrepandum, (Forsk). Revista Latino Americana de Química 39(1-2): 62-74. 
Adams TB, JB Hallagan, JM Putnam, TL Gierke, J Doull, IC Munro, P Newberne, PS Portoghese, RL Smith, BM Wagner, CS Weil, LA Woods \& A Ford. 1996. The FEMA GRAS assessment of alicyclic substances used as flavour ingredients. Food and Chemical Toxicology 34: 763-828.

Adegoke AK, WH Abdullah, MH Hakimi \& BMS Yandoka. 2015. Geochemical characterisation and organic matter enrichment of Upper Cretaceous Gongila shales from Chad (Bornu) Basin, northeastern Nigeria: Bioproductivity versus anoxia conditions. Journal of Petroleum Science and Engineering 135: 73-87.

Ahsan T, J Chen, X Zhao, M Irfan \& Y Wu. 2017. Extraction and identification of bioactive compounds (eicosane and dibutyl phthalate) produced by Streptomyces strain KX852460 for the biological control of Rhizoctonia solani AG-3 strain KX852461 to control target spot disease in tobacco leaf. AMB Express (Springer) 7: 54-63.

ATSDR. 2008. Toxicological profile for 1,1,2,2-Tetrachloroethane, 341 pp. U.S. Department of Health and Human Services, Public Health Service, Agency for Toxic Substances and Disease Registry, Atlanta.

Avio CG, S Gorbi \& F Regoli. 2016. Plastics and microplastics in the oceans: From emerging pollutants to emerged threat. Marine Environmental Research 128: 2-11.

Axelstad M, J Boberg, KS Hougaard, S Chrisiansen, PR Jacobesen, KR Mandrup, C Nellemann, SP Lund \& U Hass. 2011. Effects of pre- and postnatal exposure to the UV-filter Octyl Methoxycinnamate (OMC) on the reproductive, auditory and neurological development of rat offspring. Toxicology and Applied Pharmacology 250(3): 278-290.

Barceló D. 2003. Emerging pollutants in water analysis. TrAC, Trends in Analytical Chemistry 22(10): 14-15.

Bhagavathy S, P Sumathi \& JS Bell. 2011. Green algae Chlorococcum humicola - a new source of bioactive compounds with antimicrobial activity. Asian Pacific Journal of Tropical Biomedicine 1(1) [Suppl.]: S1-S7

Bingham E \& B Cohrssen. 2012. Trends in industrial toxicology. Patty's Toxicology 1: 1-4.

Bookstaff RC, S PaiBir, SS Bharaj, GR Kelm, RM Kulick, TK Balm \& JV Murray. 2003. The safety of the use of ethyl oleate in food is supported by metabolism data in rats and clinical safety data in humans. Regulatory Toxicology and Pharmacology 37(1): 133-148.

Borik RM. 2014. Volatile compounds extraction, fractionation and identification from the red alga Corallina officinalis. World Applied Sciences Journal 30(6): 741-746.

Brechbill GO. 2009. Arranging fine perfume compositions, 338 pp. Fragrance Books, New Jersey.

Brechbill GO. 2012. The floral notes of fragrance, $349 \mathrm{pp}$. Fragrance Books, New Jersey.

Bryan GW. 1979. Bioaccumulation of marine pollutants. Philosophical Transactions of the Royal Society B 286: 483-505.

Burn D, B Ellis, V Petrow, IA Stuart-Webb \& DM Williamson. 1957. 809. Modified steroid hormones. Part IV. 6-Methylpregnane derivatives. Journal of the Chemical Society 1957: 4092-4098.
Calvo-Flores FG, J Isac-García \& JA Dobado. 2018. Emerging pollutants: Origin, structure and properties, 518 pp. Wiley-VCH, Weinheim.

Carvalho LR, MT Fujii, NF Roque, MJ Kato \& JHG Lago. 2003. Aldingenin $A$, new brominated sesquiterpene from red alga Laurencia aldingensis. Tetrahedron Letters 44: 2637-2640.

Castillo C, H Chen, C Graves, A Maisonnasse, Y Le Conte \& E Plettner. 2012. Biosynthesis of ethyl oleate, a primer pheromone, in the honey bee (Apis mellifera L.). Insect Biochemistry and Molecular Biology 42(6): 404-416.

Chan HW, TC Lau, PO Ang, M Wu \& PK Wong. 2004. Biosorption of di(2-ethylhexyl) phthalate by seaweed biomass. Journal of Applied Phycology 16: 263-274.

Chopra AK \& C Pathak. 2010. Biosorption technology for removal of metallic pollutants-An overview. Journal of Applied and Natural Science 2(2): 318-329.

Cortés Y, E Hormazábal, H Leal, A Urzúa, A Mutis, L Parra \& A Quiroz. 2014. Novel antimicrobial activity of a dichloromethane extract obtained from red seaweed Ceramium rubrum (Hudson) (Rhodophyta: Florideophyceae) against Yersinia ruckeri and Saprolegnia parasitica, agents that cause diseases in salmonids. Electronic Journal of Biotechnology 17: 126-131.

Dokulil MT. 2003. Algae as ecological bio-indicators. In: Markert BA, AM Breure \& HG Zechmeister (eds). Bioindicators and biomonitors, pp. 285-327. Elsevier, Amsterdam.

Du X \& SH Zeisel. 2013. Spectral deconvolution for gas chromatography- mass spectrometry based metabolomics: Current status and future perspectives. Computational and Structural Biotechnology Journal 4(5): e201301013.<doi: 10.5936/csbj.201301013>

Duan P, C Hu, C Quan, T Yu, W Zhou, M Yuan, Y Shi \& K Yang. 2016. 4-Nonylphenol induces apoptosis, autophagy and necrosis in Sertoli cells: Involvement of ROS-mediated AMPK/AKT-mTOR and JNK pathways. Toxicology 341/343: 28-40.

EFSA. 2008. Flavouring Group Evaluation 25, (FGE.25): Aliphatic and aromatic hydrocarbons from chemical group 31. Scientific opinion of the panel on food additives, flavourings, processing aids and materials in contact with food. The EFSA Journal 918: 1-109.

El-Boujaddaini MN, P Haberschill \& A Mimet. 2010. Rheological behaviour and concentration distribution of paraffin slurry in horizontal rectangular channel. International Journal of Dynamics of Fluids 6(2): 145-160.

El-Demerdash E. 2011. Anti-inflammatory and antifibrotic effects of methyl palmitate. Toxicology and Applied Pharmacology 254(3): 238-244.

El-Maghraby DM. 2013. Evaluation of non- viable biomass of Laurencia papillosa for decolorization of dye waste water. African Journal of Biotechnology 12(17): 2215-2223.

El-Maghraby DM \& EM Fakhry. 2015. Lipid content and fatty acid composition of Mediterranean macro-algae as dynamic factors for biodiesel production. Oceanologia 57(1): 86-92. 
Eltz T, E Hedenström, J Bang, E Wallin \& J Andersson. 2010. $(6 R, 10 R)-6,10,14$-Trimethylpentadecan-2-one, a dominant and behaviorally active component in male orchid bee fragrances. Journal of Chemical Ecology 36(12): 1322-1326.

Fait ME, L Bakas, GL Garrote, SR Morcelle \& MCN Saparrat. 2019. Cationic surfactants as antifungal agents. Applied Microbiology and Biotechnology 103(1): 97-112.

Farré M, S Pérez, L Kantiani \& D Barceló. 2008. Fate and toxicity of emerging pollutants, their metabolites and transformation products in the aquatic environment, Trends in Analytical Chemistry 27: 991-1007.

Fasano E, F Bono-Blay, T Cirillo, P Montuori \& S Lacorte. 2012. Migration of phthalates, alkylphenols, bisphenol A and di(2-ethylhexyl)adipate from food packaging. Food Control 27(1): 132-138.

Fernandez NA, E Chacin, E Gutierrez, N Alastre, B Llamoza \& CF Forster. 1995. Adsorption of lauryl benzyl sulphonate on algae. Bioresource Technology 54: 111-115.

Fujimura T, T Kawai, M Shiga, T Kajiwara \& A Hatanaka. 1990. Long-chain aldehyde production in thalli culture of the marine green alga Ulva pertusa. Phytochemistry 29(3): 745-747.

Gadd GM. 2009. Biosorption: critical review of scientific rationale, environmental importance and significance for pollution treatment. Journal of Chemical Technology and Biotechnology 84: 13-28.

Gao D \& Z Wen. 2016. Science of the Total Environment Phthalate esters in the environment: A critical review of their occurrence, biodegradation, and removal during wastewater treatment processes. The Science of the Total Environment 541: 986-1001.

Goulitquer S, P Potin \& T Tonon. 2012. Mass spectrometrybased metabolomics to elucidate functions in marine organisms and ecosystems. Marine Drugs 10: 849-880.

Govindan M, JD Hodge, KA Brown \& M Nuñez-Smith. 1993. Distribution of cholesterol in Caribbean marine algae. Steroids 58: 178-180.

Gressler V, NS Yokoya, MT Fujii, P Colepicolo, J ManciniFilho, RP Torres \& E Pinto. 2010. Lipid, fatty acid, protein, amino acid and ash contents in four Brazilian red algae species. Food Chemistry 120(2): 585-590.

Gressler V, MT Fujii, P Colepicolo \& E Pinto. 2012. Characterization of volatile composition of Laurencia dendroidea by gas chromatography-mass spectrometry. Brazilian Journal of Pharmacognosy 22(4): 805-812.

Hatem BA, WH Abdullah, MH Hakimi \& KA Mustapha. 2016. Origin of organic matter and paleoenvironment conditions of the Late Jurassic organic-rich shales from Shabwah sub-basin (western Yemen): Constraints from petrology and biological markers. Marine and Petroleum Geology 72: 83-97.

Hay BP, DA Dixon, GJ Lumetta, R Vargas \& J Garza. 2004. Structural aspects of hydrogen bonding with nitrate and sulfate. Fundamentals and applications of anion separations, pp. 43-57. Springer, Boston.

He N, X Sun, Y Zhong, K Sun, W Liu \& S Duan. 2016. Removal and biodegradation of nonylphenol by four freshwater microalgae. International Journal of Environmental Research and Public Health 13(12): 1239. $<$ https://doi.org/10.3390/ijerph13121239>
Holt EA \& SW Miller. 2010. Bioindicators: Using organisms to measure environmental impacts. Nature Education Knowledge 3(10): 1-8.

Homans AL \& A Fuchs. 1970. Direct bioautography on thinlayer chromatograms as a method for detecting fungitoxic substances. Journal of Chromatography A 51: 327-329.

Horii Y \& K Kannan. 2008. Survey of organosilicone compounds, including cyclic and linear siloxanes, in personal-care and household products. Archives of Environmental Contamination and Toxicology 55(4): 701-710

Huang B, X Ban, J He, J Tong, J Tian \& Y Wang. 2010. Comparative analysis of essential oil components and antioxidant activity of extracts of Nelumbo nucifera from various areas of China. Journal of Agricultural and Food Chemistry 58(1): 441-448.

Inouhe M. 2005. Phytochelatins. Toxic metals in plants. Brazilian Journal of Plant Physiology 17(1): 65-78.

Islam MA, M Magnusson, RJ Brown, GAAyoko, MN Nabi \& K Heimann. 2013. Microalgal species selection for biodiesel production based on fuel properties derived from fatty acid profiles. Energies 6(11): 5676-5702.

Jiménez-Díaz I, JM Molina-Molina, A Zafra-Gómez, O Ballesteros, A Navalón, M Real, JM Sáenz, MF Fernández \& N Olea. 2013. Simultaneous determination of the UV-filters benzyl salicylate, phenyl salicylate, octyl salicylate, homosalate, 3-(4-methylbenzylidene) camphor and 3-benzylidene camphor in human placental tissue by LC-MS/MS. Assessment of their in vitro endocrine activity. Journal of Chromatography B: Analytical Technologies in the Biomedical and Life Sciences 936: 80-87.

Jobling S, T Reynolds, R White, MG Parker \& JP Sumpter. 1995. A variety of environmentally persistent chemicals, including some phthalate plasticizers, are weakly estrogenic. Environmental Health Perspectives 103(6): 582-587.

Johnson MD, NN Price \& JE Smith. 2014. Contrasting effects of ocean acidification on tropical fleshy and calcareous algae. PeerJ 2:e411. <https://doi.org/10.7717/peerj.411>

Kalhor AX, A Movafeghi, AD Mohammadi-Nassab, E Abedi \& A Bahrami. 2017. Potential of the green alga Chlorella vulgaris for biodegradation of crude oil hydrocarbons. Marine Pollution Bulletin 123: 286-290.

Kamenarska Z, A Ivanova, R Stancheva, M Stoyneva, K Stefanov, S Dimitrova-Konaklieva \& S Popov. 2006. Volatile compounds from some Black Sea red algae and their chemotaxonomic application. Botanica Marina 49(1): 47-56.

Karabay-Yavasoglu NU, A Sukatar, G Ozdemir \& Z Horzum. 2007. Antimicrobial activity of volatile components and various extracts of the red alga Jania rubens. Phytotherapy Research 21(2): 153-156.

Karley AJ, SI Powell \& JM Davies. 1997. Effect of nonylphenol on growth of Neurospora crassa and Candida albicans. Applied and Environmental Microbiology 63(4): 1312-1317.

Khan N, S Jadhav \& VK Rathod. 2016. Enzymatic synthesis of $n$-butyl palmitate in a solvent-free system: RSM optimization and kinetic studies. Biocatalysis and Biotransformation 34(3): 99-109. 
Khotimchenko SV. 2005. Lipids from the marine alga Gracilaria verrucosa. Chemistry of Natural Compounds 41(3): 285-288.

Khotimchenko SV, VE Vaskovsky \& TV Titlyanova. 2002. Fatty acids of marine algae from the Pacific Coast of North California. Botanica Marina 45(1): 17-22.

Kim S, J Chen, T Cheng, A Gindulyte, J He, S He, Q Li, BA Shoemaker, PA Thiessen, B Yu, L Zaslavsky, J Zhang \& EE Bolton. 2019. PubChem 2019 update: Improved access to chemical data. Nucleic Acids Research 47(D1): 1102-1109.

Knaggs EA, JA Yeager, L Varenyi \& E Fischer. 1965. Alpha sulfo fatty esters in biologically soft detergent formulations. Journal of the American Oil Chemists Society 42(9): 805-810.

Koch HM, R Preuss \& J Angerer. 2006. Di(2-ethylhexyl) phthalate (DEHP): Human metabolism and internal exposure - An update and latest results. International Journal of Andrology 29(1): 155-165.

Kolkwitz R \& M Marsson. 1908. Ökologie der tierische Saprobien. Beiträige zur Lehre von der biologische Gewässerbeurteilung. International Review of Hydrobiology 2: 126-152.

Lalko J, A Lapczynski, D McGinty, S Bhatia, CS Letizia \& AM Api. 2007. Fragrance material review on $\beta$-ionone. Food and Chemical Toxicology 45(1): 241-247.

Lee YC, HH Chang \& CH Lui. 2010. Methyl palmitate: A potent vasodilator released in the retina. Investigative Ophthalmology and Visual Science 51(9): 4746-4753.

Lotfy MM, HM Hassan, MH Hetta, AO El-Gendy \& R Mohammed. 2018. Di-(2-ethylhexyl) Phthalate, a major bioactive metabolite with antimicrobial and cytotoxic activity isolated from River Nile derived fungus Aspergillus awamori. Beni-Suef University Journal of Basic and Applied Sciences 7(3): 263-269.

Lui JQ, JM Wu, XL Kou \& Q Hong. 2008. Studies on chemical constituents from Grewia biloba. Journal of Chinese medicinal material 31(10): 1505-1507.

Mackintosh CE, J Maldonado, J Hongwu, N Hoover, A Chong, MG Ikonomou \& FAPC Gobas. 2004. Distribution of phthalate esters in a marine aquatic food web: comparison to polychlorinated biphenyls. Environmental Science \& Technology 38: 2011-2020.

Magi E \& M Di Carro. 2016. Marine environment pollution: the contribution of mass spectrometry to the study of seawater. Mass Spectrometry Reviews 37(4): 492-512.

Martín J, A Zafra-Gómez, F Hidalgo, AJ Ibáñez-Yuste, E Alonso \& JL Vilchez. 2017. Multi-residue analysis of 36 priority and emerging pollutants in marine echinoderms (Holothuria tubulosa) and marine sediments by solid-liquid extraction followed by dispersive solid phase extraction and liquid chromatography-tandem mass spectrometry analysis. Talanta 166: 336-348.

Marx CE, DW Bradford, RM Hamer, JC Naylor, TB Allen, JA Lieberman, JL Strauss \& JD Kilts. 2011. Pregnenolone as a novel therapeutic candidate in schizophrenia: Emerging preclinical and clinical evidence. Neuroscience 191: 78-90.
McInnes AG, JA Walter \& LC Wright. 1979. Biosynthesis of hydrocarbons by algae: decarbosylation of stearic acid to $\mathrm{n}$-heptadecane in Anacystis nidulans determined by ${ }^{13} \mathrm{C}$ and ${ }^{2} \mathrm{H}$-labeling and ${ }^{13} \mathrm{C}$ NMR. Lipids 15(9): 609-615.

Mearns AJ, DJ Reish, PS Oshida, T Ginn, MA RempelHester, C Arthur, N Rutherford \& R Pryor. 2015. Effects of pollution on marine organisms. Water Environment Research 87(10): 1718-1816.

Memić M, A Selović \& O Mahmutović. 2011. Toxicity of chlorinated phenols against ligninolytic fungi Hypoxylon fragiforme and Coniophora puteana. Works of the Faculty of Forestry University of Sarajevo 2: 31-44.

Millero FJ, R Feistel, DG Wright \& TJ McDougall. 2008. The composition of standard seawater and the definition of the reference-composition salinity scale. Deep-Sea Research Part I 55: 50-72.

Moreau J, D Pesando, P Bernard, B Caram \& JC Pionnat. 1988. Seasonal variations in the production of antifungal substances by some Dictyotales (brown algae) from French Mediterranean coast. Hydrobiology 162: 157-162.

Mortimer JV \& LA Luke. 1966. The determination of normal paraffins in petroleum products. Analytica Chimica Acta 38: 119-126.

Moustafa AMY, A Gehan, GA El-Shoubaky \& EAE Salem. 2008. Comparative phycochemical investigation of hydrocarbons content on some marine seaweeds algae. Research Journal of Phytochemistry 2: 10-17.

Moustafa MFM, SA Alamri, TH Taha \& SA Alrumman. 2013. In vitro antifungal activity of Argemone ochroleuca Sweet latex against some pathogenic fungi. African Journal of Biotechnology 12(10): 1132-1137.

Nazzaro F, F Fratianni, R Coppola \& V De Feo. 2017. Essential oils and antifungal activity. Pharmaceuticals 10(4): 86. <https://doi.org/10.3390/ph10040086>

Noguchi T, M Ikawa, JJ Uebel \& KKAndersen. 1979. Lipid constituents of the red alga Ceramium rubrum. A search for antimicrobial and chemical defense substances. In: Hoppe HA, T Levring \& Y Tanaka (eds). Marine algae in pharmaceutical Science, pp. 711-719. Walter de Gruyter, Berlin.

Oliveira ALL, DB Silva, NP Lopes, HM Debonsi \& NS Yokoya. 2012. Chemical constituents from red algae Bostrychia radicans (Rhodomelaceae): new amides and phenolic compounds. Química Nova 35(11): 2186-2188.

Olofsson P, M Hultqvist, L Hellgren \& R Holmdahl. 2014. Phytol: a chlorophyll component with anti-inflammatory and metabolic properties. In: Jacob C, G Kirsch, AJ Slusarenko, PG Winyard \& T Burkholz (eds). Recent advances in redox active plant and microbial product, $\mathrm{pp}$. 345-359. Springer, Dordrecht.

Omar WMW. 2010. Perspectives on the use of algae as biological indicators for monitoring and protecting aquatic environments, with special reference to Malaysian freshwater ecosystems. Tropical Life Sciences Research 21(2): 51-67.

Osman MEH, AM Aboshady \& ME Elshobary. 2013. Production and characterization of antimicrobial active substance from some macroalgae collected from AbuQir bay (Alexandria) Egypt. African Journal of Biotechnology 12(49): 6847-6858. 
Ouellette RJ \& JD Rawn. 2015. Carboxylic acids and esters. Principles of Organic Chemistry 20: 287-314.

Paluch E, A Piecuch, E Oblak, L Lamch \& KA Wilk. 2018. Antifungal activity of newly synthesized chemo degradable dicephalic-type cationic surfactants. Colloids and Surfaces B: Biointerfaces 164(1): 34-41.

Paoloni L, A Patti \& F Mangano. 1975. The hydrogen bond with carbonyl groups: Theoretical study of the correlation between the X-H stretching frequency shift and the $\mathrm{C}=\mathrm{O}$ group properties. Journal of Molecular Structure 27: 123137.

Parris N, JK Weil \& WM Linfield. 1973. Soap based detergent formulations. V. Amphoteric lime soap dispersing agents. Journal of the American Oil Chemists' Society 50(12): 509-512.

Pereira H, L Barreira, F Figueiredo, L Custódio, C Vizetto-Duarte, C Polo, E Resek \& J Varela. 2012. Polyunsaturated fatty acids of marine macroalgae: Potential for nutritional and pharmaceutical applications. Marine Drugs 10(9): 1920-1935.

Peres JCF, LR de Carvalho, E Gonçales, LOS Berian \& JD Felicio. 2012. Evaluation of antifungal activity of seaweed extracts. Ciência e Agrotecnologia 36(3): 294-299.

Phaechamud T \& C Savedkairop. 2012. Biological and chemical sciences contact angle and surface tension of some solvents used in pharmaceuticals. Research Journal of Pharmaceutical 3(4): 513-529.

Plaza M, S Santoyo, L Jaime, GR García-Blairsy, M Herrero, FJ Señoráns \& E Ibañez. 2010. Screening for bioactive compounds from algae. Advanced Biofuels and Bioproducts 51: 833-872.

Plouguerné E, H Kikuchi, Y Oshima, E Deslandes \& V Stiger-Pouvreau. 2006. Isolationof Cholest-5-en-3-ol formate from the red alga Grateloupia turuturu Yamada and its chemotaxonomic significance. Biochemical Systematics and Ecology 34: 714-717.

Poon R, P Lecavalier, R Mueller, V Valli, B Procter \& I Chu. 1997. Subchronic oral toxicity of di-n-octyl phthalate and di(2-ethylhexyl) phthalate in the rat. Food and Chemical Toxicology 35(2): 225-239.

Rahalison L, M Hamburger, M Monod, E Frenk \& K Hostettmann. 1994. Antifungal tests in phytochemical investigations comparison of bioautographic methods using phytopatogenic and human pathogenic fungi. Planta Medica 60: 41-44

Rajfur M. 2014. Algae - heavy metals biosorbent. Ecological Chemistry and Engineering S 20(1): 23-40.

Rajfur M \& A Klos. 2014. Use of algae in active biomonitoring of surface waters. Ecological Chemistry and Engineering S 21(4):561-576.

Rezanka T, J Zahradník \& M Podojil. 1982. Hydrocarbons in green and blue-green algae. Folia Microbiologica 27(6): 450-454.

Rietschel RL \& CW Lewis. 1978. Contact dermatitis to homomenthyl salicylate. Archives of Dermatology 114(3): 442-443.

Rowdhwal SSS \& J Chen. 2018. Toxic effects of Di-2-ethylhexyl Phthalate: An overview. BioMed Research International 1-10. 2018:1750368. <doi: 10.1155/2018/1750368. eCollection 2018>
Sahu A, I Pancha, D Jain, C Paliwal, T Ghosh, S Patidar, S Bhattacharya \& S Mishra. 2013. Fatty acids as biomarkers of microalgae. Phytochemistry 89: 53-58.

Sambandan DR \& D Ratner. 2011. Sunscreens: An overview and update. Journal of the American Academy of Dermatology 64(4): 748-758.

Schaeffer L. 2008. The role of functional groups in drugreceptor interactions. The practice of medicinal chemistry, pp. 359-378. Elsevier, Amsterdam.

Schlumpf M, P Schmid, S Durrer, M Conscience, K Maerkel, M Henseler, M, Gruetter, I Herzog, S Reolon, R Ceccatelli, O Faass, E Stutz, H Jarry, W Wuttke \& W Lichtensteiger. 2004. Endocrine activity and developmental toxicity of cosmetic UV filters -an update. Toxicology 205: 113-122.

Sharanagat VS, V Singla \& L Singh. 2019. Bioactive compounds from marine sources. In: Goyal MG, HAR Suleria \& S Kirubanandan (eds). Technological processes for marine foods, from water to fork: bioactive compounds, industrial applications and genomics, pp. 23-42. Apple Academic Press, Toronto.

Shobier AH, SAA Ghani \& KM Barakat. 2016. GC/ MS spectroscopic approach and antifungal potential of bioactive extracts produced by marine macroalgae. The Egyptian Journal of Aquatic Research 42(3): 289-299.

Shore RN \& WB Shelley. 2015. Stearyl alcohol and propylene in fluocinonide cream glycol. Archives of Dermatology 109: 397-399.

Sieppi E, K Vähäkangas, A Rautio, F Ietta, L Paulesu \& P Myllynen. 2016. The xenoestrogens, bisphenol A and para-nonylphenol, decrease the expression of the ABCG2 transporter protein in human term placental explant cultures. Molecular and Cellular Endocrinology 429: 41-49.

Sousa GS \& ALM Cocentino. 2004. Macroalgas como indicadoras da qualidade ambiental da Praia de Piedade, PE. Tropical Oceanography 32: 1-22.

Sousa JCG, AR Ribeiro, MO Barbosa, MFR Pereira \& AMT Silva. 2018. A review on environmental monitoring of water organic pollutants identified by EU guidelines. Journal of Hazardous Materials 344: 146-162.

Souza NJ de \& WR Nes. 1969. Presence of phytol in brown and blue-green algae and its relationship to evolution. Phytochemistry 8: 819-822.

Stein EM, P Colepicolo, FAK Afonso \& MT Fujii. 2011. Screening for antifungal activities of extracts of the Brazilian seaweed genus Laurencia (Ceramiales, Rhodophyta). Revista Brasileira de Farmacognosia 21: 290-295.

Stranska-Zachariasova M, I Kurniatanty, H Gbelcova, M Jiru, J Rubert, TGT Nindhia, CW D'Acunto, SH Sumarsono, MI Tan, J Hajslova \& T Ruml. 2017. Bioprospecting of Turbinaria macroalgae as a potential source of health protective compounds. Chemistry \& Biodiversity 14: e1600192. <doi: 10.1002/ cbdv.201600192>

Sugisawa H, K Nakamura \& H Tamura. 1990. The Aroma profile of the volatiles in marine green algae (Ulva pertusa). Food Reviews International 6(4): 573-589. 
Usov AI. 2011. Polysaccharides of the red algae. Advances in carbohydrate chemistry and biochemistry 65: 115-217.

Uyanik A, A Öktemer \& E Loğoğlu. 2009. Antimicrobial and antifungal activity study of poly substituted benzene derivative. Communications Faculty of Sciences University of Ankara, Series B 55(1): 17-22.

Vazquez D \& GA Mansoori. 2000. Identification and measurement of petroleum precipitates. Journal of Petroleum Science and Engineering 26: 49-55.

Vimala T \& TV Poonghuzhali. 2017. In vitro activity of solvent extracts of marine brown alga, Hydroclathrus clathratus (C. Agardh) M. Howe from of golf of Mannar. Journal of Applied Pharmaceutical Science 7: 157-162.

Widianingsih D, R Hartati, H Endrawati \& J Mamuaja. 2012. Fatty acid composition of marine microalgae in Indonesia. Journal of Tropical Biology and Conservation 10: $75-82$.
Xian Q, H Chen, H Liu, H Zou \& D Yin. 2006. Isolation and identification of antialgal compounds from the leaves of Vallisneria spiralis L. by activity-guided fractionation. Environmental Science and Pollution Research International 13(4): 233-237.

Yamamoto M, S Baldermann, K Yoshikawa, A Fujita, $\mathbf{N}$ Mase \& N Watanabe. 2014. Determination of volatile compounds in four commercial samples of Japanese green algae using solid phase microextraction gas chromatography mass spectrometry. Scientific World Journal 289780: 8. <doi: 10.1155/2014/289780>

Yao S, M Johannsen, RG Hazell \& KA Jorgensen. 1998. Total synthesis of $(\mathrm{R})$-dihydroactinidiolide and $(\mathrm{R})$ -actinidiolide using asymmetric catalytic hetero-diels-alder methodology. Journal of Organic Chemistry 63(1): 118121. <doi: $10.1021 /$ jo $971528 \mathrm{y}>$

Zielińska A \& I Nowak. 2014. Fatty acids in vegetable oils and their importance in cosmetic industry. Chemik 68(2): $103-110$.

Received 5 June 2019 and accepted 28 June 2020

Editor: Claudia Bustos D. 